\section{AMBIDEXTROUS \\ AND PNEUMATIC}

The ambidextrous Anthos R7 is the ideal choice of highly adaptable, modular Treatment Centre and is available from your premier R7 distributor Henry Schein Minerva.

The variety of $\mathrm{R} 7$

configurations means the chair makes effective use of available surgery space and in just a few simple moves can be converted to either a righthanded or left-handed unit, making it compatible to all user requirements.

The focal point of the Anthos R7 is its true versatility and array of features to meet the requirements for comfort, control and hygiene. The control panel's colour display allows the operator to visualise handpiece settings for speed, torque and endo in real time, whilst the pneumatic triple axis headrest and chair swivel option enable good access when working with the microscope or surgical trolley. Combine this with triple axis operating light and users receive a greater view of the oral cavity.

The R7 can be completely controlled from the dentist's element, nurse's element, the chair itself or from the multifunction foot control which allows handpiece speed regulation, chair adjustments and chair programme access for a completely hands-free operation.

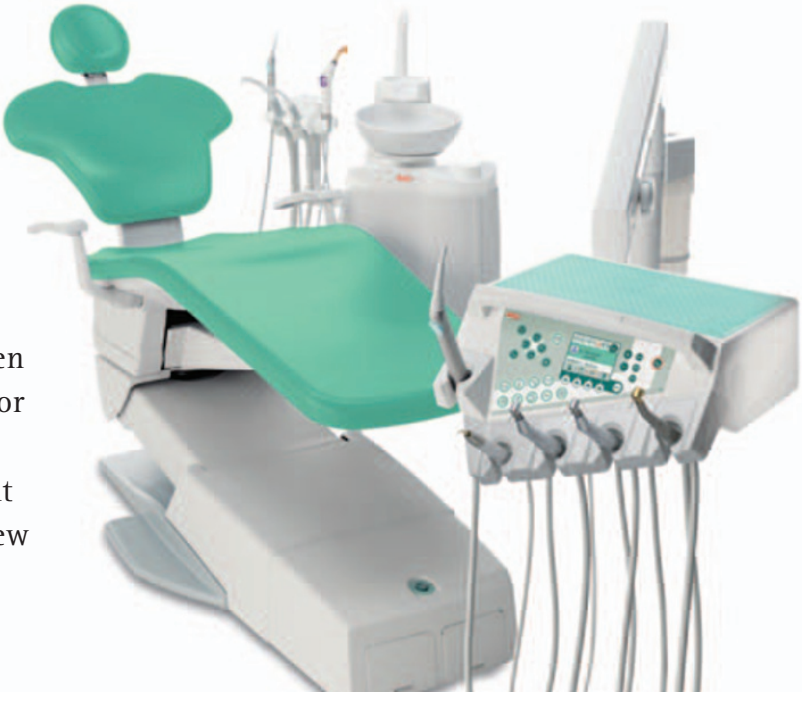

Additionally, the treatment centre comes pre-wired to offer compatibility with the Anthos Camera and Screen system at any time in the future.

For a showroom appointment in London, Leeds, Glasgow or Cardiff, call 08700102041 or email sales@henryschein.co.uk.

\section{VISIT THE COURTYARD OF DREAMS}

Sident Dental Systems offer the most comprehensive bespoke project management service for surgery refurbishments to make your dreams a reality.

Sident offers a choice from the complete range of Sirona Treatment Centres, 2D and 3D digital and film based X-ray apparatus - including Galileos 3D digital cone-beam equipment their extensive range of Sirona handpieces, and auxiliary items including SiroLaser Advance, SIROEndo and the DAC Universal sterilisation unit. To support these, they offer DURR Suction and Dental Art Cabinetry, as well as other essential equipment from similarly tried and trusted manufacturers.

Wherever possible, clients are invited to visit The Courtyard Clinic, Sident's state-of-the-art training and showroom facility, where they can see the complete range in action. Clients will then be able to identify the best solution for their individual needs, allowing Sident's Design Specialists to incorporate their desires into the most appropriate and ergonomically efficient layout.

Sident will undertake a complete project management service, including installation and post installation service support.

For further information call Sident Dental Systems on 01932582900 or email j.colville@ sident.co.uk.

\section{TANGIBLE PROOF OF ACCESSIBILITY}

L S Browne Technical Services has launched Simply CADCAM, a range of core restorative products created using CADCAM to deliver the perfect combination of high quality and great value. Simply CADCAM is designed to help practices become more competitive as the range includes restorations in metal and ceramic for both nonimplant and implant borne units and offers the dual benefits of predictability and clinical accuracy without the need for costly investment by the practitioner.

For the practitioner, the process is simple and straightforward as it requires no change in technique and no in-surgery equipment needs to be purchased. The CAD technology is at the laboratory - where a specifically trained technician works with the latest generation software to design the restoration exactly to the dentist's prescription.

Simply CADCAM is tangible proof that L S Browne Technical Services has become even more accessible to all, and first and foremost, is dedicated to providing excellent quality dentistry.

For more information about Simply CADCAM contact L S Browne Technical Services on 02083911188.

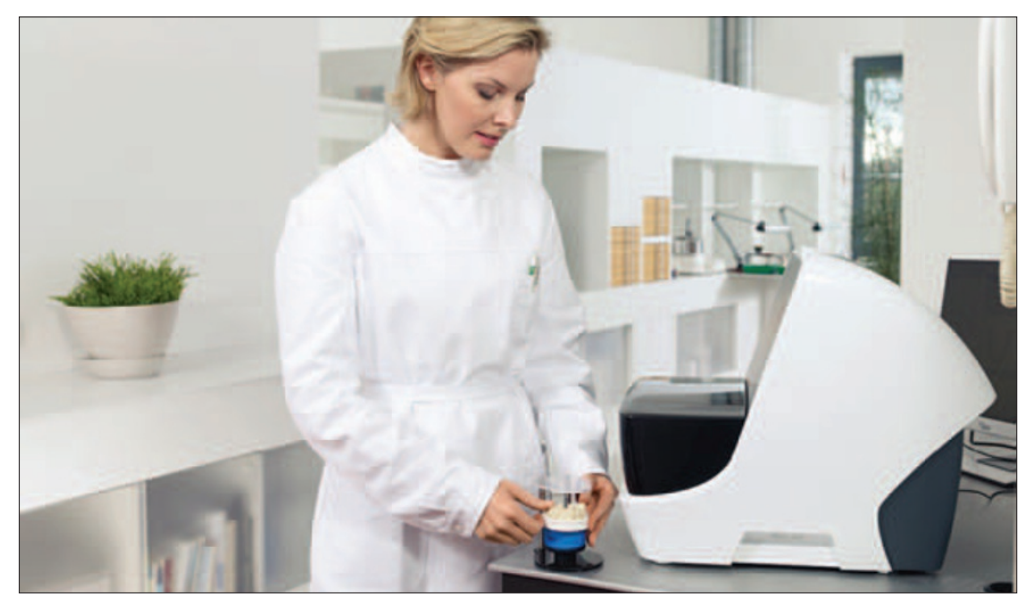

